\title{
CHALLENGES IN CLASSIFYING ISCHEMIC STROKE
}

\author{
Sofie A. Simonsen, $M D^{1}$, Anders S. West, $M D^{1}$, Adam V. Andersen, $M D^{1}$, Frauke Wolfram, $\mathrm{MD}^{2}$, \\ Poul Jennum MD, DMSc ${ }^{3}$, Helle K. Iversen MD, DMSc ${ }^{1}$
}

Rigshospitalet

${ }^{1}$ Clinical Stroke Research Unit, Department of Neurology; ${ }^{2}$ Department of Radiology;

${ }^{3}$ Danish Center for Sleep Medicine. Rigshospitalet, University of Copenhagen.

\section{BACKGROUND}

The TOAST (Trial of Org 101072 in Acute Stroke Treatment) classification is most frequently used to categorize the etiology of index ischemic strokes but does not reflect the heterogeneous vascular pathophysiology in the patient. The current aim was to assess the presence of small and large vessel disease across TOAST groups.

\section{METHODS}

Consecutive admitted acute ischemic stroke patients were classified according to TOAST into large artery atherosclerosis (LAA), cardioembolism (CE), small-vessel occlusion (SVO), other determined etiology and undetermined etiology. The total small vessel disease (SVD) score (0-4) was calculated based on cerebral MRI. The assessment was supplemented with ankle brachial index $(A B I)$, estimated glomerular filtration rate (eGFR) and peripheral reactive hyperemia index $(\mathrm{RHI})$.

\section{RESULTS}

We included 99 ischemic stroke patients, median age 68 years (range 36-88), 44\% women, NIHSS 2 (range 016).

Carotid stenosis and atrial fibrillation (AF) were associated with the LAA $(p=0.023)$ and CE $(p<0.001)$
TOAST groups, respectively. We found no association between SVD score and the SVO TOAST group $(p=0.59)$. RHI $(p=0.39), \quad A B I \quad(p=0.20)$, creatinine $(p=0.31)$, and eGFR $(p=0.79)$ also were not associated with TOAST groups.

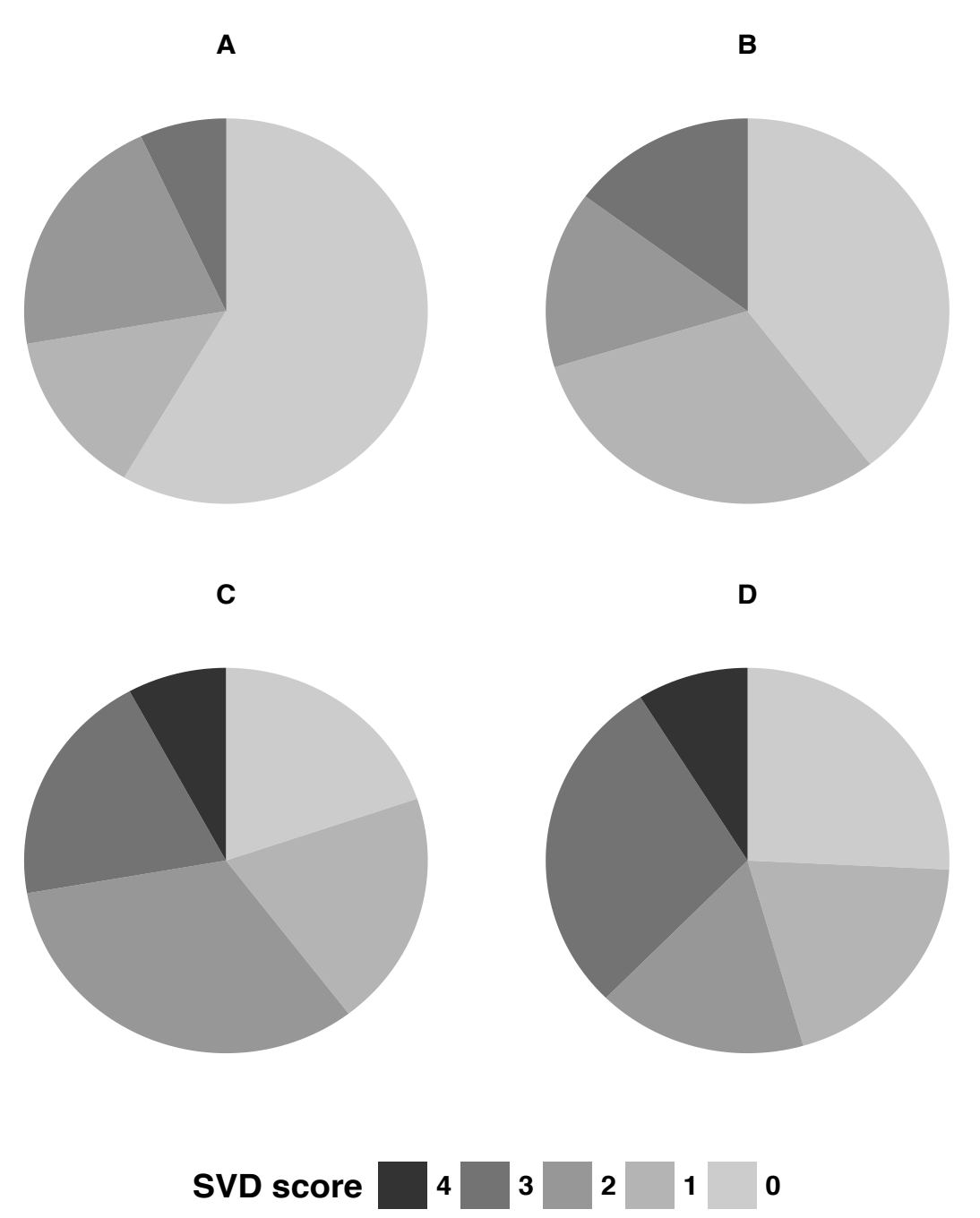

Figure 1 Distribution of the SVD score in TOAST groups. A: Large artery atherosclerosis; B: cardioembolism; C: small vessel occlusion; D: undetermined etiology.
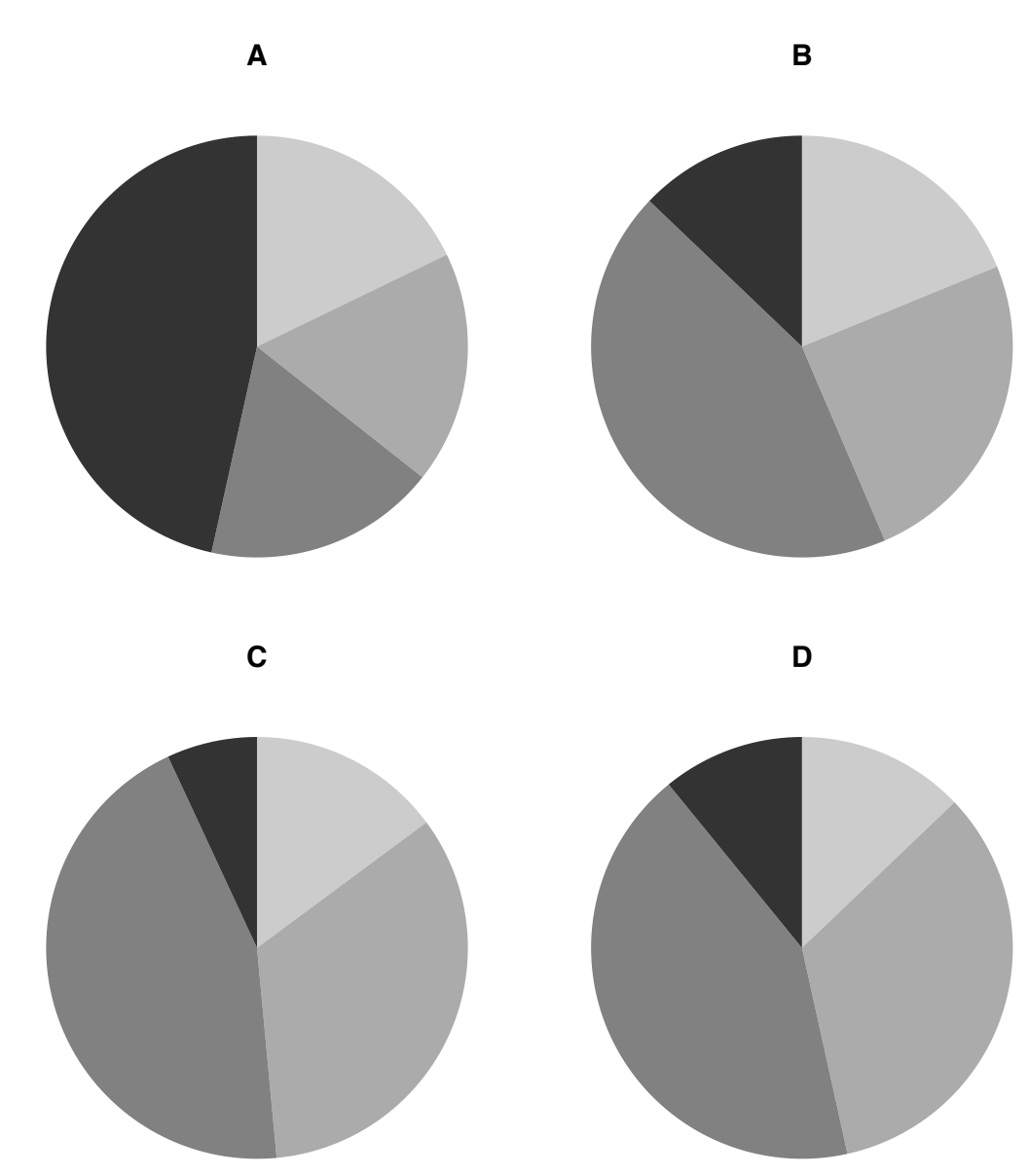

Atherosclerosis

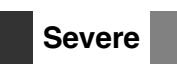

Figure 2 Distribution of internal carotid artery atherosclerosis in TOAST groups. A: Large artery atherosclerosis; B: cardioembolism; C: small vessel occlusion; D: undetermined etiology.

\section{CONCLUSION}

The presence of cerebral SVD and peripheral large and small vessel disease ( $A B I, R H I$, and eGFR) are independent of the TOAST classification. Carotid stenosis and $A F$ are associated with their relevant TOAST groups, as expected. A prerequisite for exploring stroke mechanisms is knowledge of the pathophysiology being investigated. Therefore, an operational classification system including the presence of all stroke pathophysiologies in the individual patient is strongly needed. 